\title{
BMJ Open Telemedicine-guided education on secondary stroke and fall prevention following inpatient rehabilitation for Texas patients with stroke and their caregivers: a feasibility pilot study
}

\author{
Mansi M Jhaveri, ${ }^{1}$ Ruby Benjamin-Garner, ${ }^{2}$ Nahid Rianon, ${ }^{3}$ Mark Sherer, ${ }^{4}$ \\ Gerard Francisco, ${ }^{5}$ Farhaan Vahidy, ${ }^{6}$ Kayta Kobayashi, ${ }^{7}$ Mary Gaber, ${ }^{8}$ \\ Paige Shoemake, ${ }^{9}$ Kim Vu, ${ }^{10}$ Alyssa Trevino, ${ }^{11}$ James Grotta, ${ }^{6}$ Sean Savitz ${ }^{6}$
}

To cite: Jhaveri MM, Benjamin-Garner R, Rianon N, et al. Telemedicine-guided education on secondary stroke and fall prevention following inpatient rehabilitation for Texas patients with stroke and their caregivers: a feasibility pilot study. BMJ Open 2017;7:e017340. doi:10.1136/ bmjopen-2017-017340

- Prepublication history for this paper is available online. To view, these files please visit the journal online (http://dx.doi. org/10.1136/bmjopen-2017017340).

Received 17 April 2017 Revised 13 June 2017 Accepted 20 July 2017

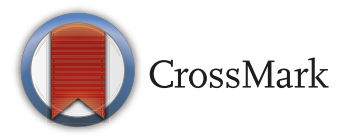

For numbered affiliations see end of article.

Correspondence to

Dr Mansi M Jhaveri;

mansi.m.jhaveri@uth.tmc.edu

\section{ABSTRACT}

Introduction The aftermath of stroke leaves many consequences including cognitive deficits and falls due to imbalance. Stroke survivors and families struggle to navigate the complex healthcare system with little assistance posthospital discharge, often leading to early hospital readmission and worse stroke outcomes. Telemedicine Guided Education on Secondary Stroke and Fall Prevention Following Inpatient Rehabilitation feasibility study examines whether stroke survivors and their caregivers find value in telerehabilitation (TR) home visits that provide individualised care and education by a multidisciplinary team after discharge from inpatient rehabilitation.

Methods and analysis A prospective, single arm, pilot study is designed to evaluate the feasibility of weekly TR home visits initiated postdischarge from inpatient rehabilitation. Newly diagnosed patients with stroke are recruited from a Houston-based comprehensive stroke centre inpatient rehabilitation unit, loaned an iPad with data plan and trained to use information technology security-approved videoconferencing application. After hospital discharge, six weekly TR home visits are led by rotating specialists (pharmacist, physical/occupational therapist, speech therapist, rehabilitation physician, social worker, geriatrician specialised in fracture prevention) followed by satisfaction survey on week 7 . Specialists visually assess patients in real time, educate them on secondary stroke and fall prevention and suggest ways to improve function including direct medical interventions when indicated. Primary outcomes are proportion of eligible patients consenting to the study, participation rate in all six TR home visits and satisfaction score. The study started 31 December 2015 with plan to enrol up to 50 patients over 24 months. Feasibility study results will inform us as to whether a randomised controlled trial is warranted to determine efficacy of TR home visit intervention in improving stroke outcomes.

Ethics and dissemination Ethics approval obtained by the Institutional Review Board (IRB), Committee for the Protection of Human Subjects, IRB number: HSC-MS-14-0994. Study results will be submitted for publication in a peer-reviewed journal.
Strengths and limitations of this study

- The study incorporates a multidisciplinary team of specialists who have the opportunity to intervene on medical problems for stroke rehabilitation patients discharged to the community.

- The study population includes underserved stroke rehabilitation patients who may be uninsured, living in rural locations and Spanish speaking with limited access to healthcare resources.

- The study uses an information technology securityapproved videoconferencing application and conducts all telerehabilitation home visits in a private setting to protect patient confidentiality.

- The videoconferencing application may be difficult for older or cognitively impaired stroke patients to use and may require caregiver assistance.

- The videoconferencing technology may not work in rural locations with poor reception.

\section{BACKGROUND}

Stroke survivors and their caregivers tackle numerous barriers after hospital discharge including cognitive/physical disability, rotation of caregivers, social isolation, lack of socioeconomic resources and geographical constraints which may limit their access to follow-up healthcare and put patients at risk for further disability and hospital readmission. ${ }^{12}$ Despite the expansive manpower and resources invested, $14 \%$ of patients with ischaemic stroke discharged from the hospital are readmitted within 30 days. ${ }^{3}$ According to a randomised controlled trial on a postdischarge follow-up service for stroke survivors, hospital readmission is common, and follow-up intervention after discharge is a way to prevent readmission for patients, particularly in those with long inpatient 
rehabilitation. ${ }^{1}$ However, under the present system, we have not developed enough transitional care programme that address the barriers preventing community stroke survivors from achieving their highest potential.

One approach to fill this gap during the critical period between patient discharge from inpatient rehabilitation programme to community is telerehabilitation (TR) home visits. Various forms of telemedicine exist including automated systems that trigger alerts, mobile apps and videoconferencing sessions with 1:1 contact with a healthcare provider. The purpose of these various forms of telemedicine is to increase access to healthcare resources for patients who are often located at a distance from a hub medical centre and cannot travel to visit their healthcare provider in person. Previous telemedicine studies to improve transitional care have been conducted in diabetes, ${ }^{4}$ renal transplant ${ }^{5}$ and heart failure populations $^{6}$ and have shown promising results including a $21 \%$ reduction in hospital readmission rate for remotely monitored heart failure patients. Telemonitoring has also been used in the stroke population to improve lower extremity strength in veterans up to 2 years poststroke, ${ }^{7}$ reduce depression and strain on stroke caregivers ${ }^{8}$ and incorporate telemedicine home-based rehabilitation in patients with stroke up to 2 years poststroke. ${ }^{9}$ However, there are currently no transitional care telemedicine education home programme focused on directly intervening with stroke rehabilitation patients immediately after discharge from inpatient rehabilitation facility.

Telemedicine Guided Education on Secondary Stroke and Fall Prevention Following Inpatient Rehabilitation (TM-SAFER) feasibility study brings the team of rehabilitation specialists to the patient's home immediately after hospital discharge to empower stroke survivors. By weekly communication via videoconferencing application on iPad, patients have the opportunity to receive individualised care with different rehabilitation specialists including pharmacist, physical/occupational therapist, speech therapist, rehabilitation physician, social worker and geriatrician specialised in fracture prevention. These are specialists who may have been involved in the patient's care while in the hospital and can provide continued follow-up of medical issues, and when required, direct medical interventions to prevent events that could lead to hospital readmission. The primary purpose of this feasibility pilot study is to determine if stroke survivors are able to participate in TR home visits immediately posthospital discharge, and whether stroke survivors and their families find these sessions valuable. We are seeking to determine what portion of persons with stroke would consent to this type of study. We are also evaluating what portion of patients have wireless reception of sufficient quality to support the TR home visits. We are examining whether this novel videoconferencing technology is accessible for the typical stroke population. This older population may have more limited experience with iPad or similar technologies than the general population. They are likely to have cognitive, motor and/or sensory deficits which may complicate their ability to use this technology. They may view TR home visits as intrusive. We seek to determine what portion of participants (persons with stroke and caregivers) believe that TR home visits increase access to healthcare resources, intercept medical problems early on, save time and money, protect confidentiality and offer patients/caregivers the opportunity to ask questions that are specific to their recovery.

\section{OBJECTIVES}

\section{Primary objectives}

This prospective feasibility study is evaluating several feasibility objectives, including the proportion of eligible patients who consent to the study, patient compliance and satisfaction with a TR home visit intervention led by a multidisciplinary team in the first 6 weeks after discharge from inpatient rehabilitation. We hypothesise that the majority of eligible patients approached for the study will consent, and that at least $70 \%$ of participants will complete all six TR home visits. Additionally, we anticipate that $80 \%$ of participants will report satisfaction with TR home visits at the level of $70 \%$ or more. Our goal is to provide valuable transitional rehabilitation care for stroke survivors through weekly contact with specialty rehabilitation providers and identify various reasons patients may or may not find the study intervention beneficial.

\section{Secondary objectives}

This feasibility study will provide important data for sample size estimates and inform on the logistics for a future randomised controlled trial. We plan to observe per cent medication refill, aspiration risk via swallow evaluation, ${ }^{10}$ Montreal Cognitive Assessment (MoCA) score ${ }^{11}$ and change in Patient Health Questionnaire (PHQ9) Depression Screen Scores, ${ }^{12}$ Falls Self Efficacy Scale Scores, ${ }^{13}$ Reintegration to Normal Living Index Scores, ${ }^{14}$ and Fracture Risk Assessment Tool (FRAX) Scores ${ }^{15}$ to assess fracture risk in patients participating in the study. We will also observe changes in medical care plan, primary care physician follow-up, number of falls, fall-related injuries, recurrent strokes, emergency department (ED) visits and hospital readmissions.

\section{METHODS}

This is a prospective, feasibility pilot study conducted at a single site from 31 December 2015-31 December 2017. Patients with stroke admitted to an inpatient rehabilitation department of a Houston Comprehensive Stroke Center are recruited by the principal investigator (PI) and/or bilingual research coordinator The PI and/or bilingual research coordinator will obtain informed consent from either patient or, if patient is unable to consent due to cognitive impairment, patient's caregiver. Total number of patients eligible for study who refuse to consent and reasons for refusal will be recorded. The enrolled study patients and their caregivers will be loaned an iPad with 
data plan and instructed in the use of information technology (IT)-approved videoconferencing application. This application includes built-in security to maintain patient confidentiality, and also allows us the ability to speak with and visually assess patients with stroke in their home for problems including speech difficulty, arm or leg weakness or gait disturbance. All videoconference calls are conducted in a private office setting to protect patient information. Participants will be given advance notification of their weekly TR home visit appointment times at discharge as well as complimentary medication pill box organiser. We will monitor compliance with participation in each TR home visit. Following six TR home visits, patients with stroke and/or caregivers will be surveyed to assess programme acceptability and satisfaction with care. At the end of the study, participants are asked to return the iPad via provided preaddressed stamped envelope. We expect the study to last 24 months.

\section{Eligibility criteria}

This study will be enrolling patients from an inpatient rehabilitation unit of a Houston comprehensive, certified stroke centre. A large percentage of our patient population includes uninsured, Spanish speaking, and rural patients who are particularly underserved. These patients have a higher rate of stroke and poorer post-stroke outcomes.

Entry criteria are structured to enrol adult male and female patients with newly diagnosed ischaemic stroke or mixed ischaemic stroke, requiring assistance in at least one Activity of Daily Living (ADL) at the time of enrolment, with caregiver support and who were previously independent, living in the community as Texas resident, and English and/or Spanish speaking. Full, detailed study inclusion and exclusion criteria are shown in figure 1.

\section{Intervention: TR home visit}

Participants enrolled in the study will receive weekly TR home visits by a team of rehabilitation specialists who may have been involved in the care of the patient during their hospital stay. The benefit this team may provide is seamless continuity of care and more efficient evaluation of follow-up issues. The order of contact by specialists is based on what we consider to be the most critical for preventing recurrent stroke, fall related injuries, and other secondary complications. During the course of the study, a revision was made to have the ability to change the order of calls based on patient's needs. Full detailed study intervention scheme is shown in figure 2.

On week 1 , the pharmacist will use videoconference to visually assess the patient's ability to set up a medication pill box organiser, per cent medications refilled and reasons for non-compliance including medication cost or side effects. The pharmacist will also recommend generic or alternate medications as indicated and educate patients/caregivers on the importance of antiplatelet medications and 3-hydroxy-3-methylglutaryl-coenzyme A reductase inhibitors for secondary stroke prevention. On week 2, physical/occupational therapists will use videoconferencing to visually assess patients in the home environment, implement strategies to reduce fall risks such as adjusting equipment and administer a falls self-efficacy scale (assess fear of falling) and a reintegration to normal living index (assess patient quality of life). On week 3 , a speech therapist will use visual information to identify choking, coughing, delayed swallow to assess risk for aspiration pneumonia as well as administer cognitive screening via the MoCA score. On week 4, the stroke rehabilitation physician will visually examine patient via videoconference and identify signs of recurrent stroke, fall related injury, provide assessment of common factors leading to falls including urinary tract infection, pneumonia, deep venous thrombosis and seizure. Stroke rehabilitation physician will assess for stability or improvement in blood pressure, blood sugar and other laboratory values through patient reports and medical record review. On week 5, the social worker will assess whether patients/ caregivers received education on programme for the indigent, went to doctor/therapy appointments and obtained all necessary equipment. In addition, the social worker will assess the patient for depression via PHQ9 depression screen. On week 6 , a geriatrician with specialty training in osteoporosis and fracture prevention will administer the FRAX questionnaire for assessment and offer recommendations on prevention of bone fractures. On week 7, an epidemiologist will administer a patient satisfaction survey. The survey will assess whether we addressed patient's/caregiver's concerns, if the intervention saved patients time and money and identify if patients had any falls, new strokes, ED visits or readmission to the hospital. Epidemiologist will also administer falls self-efficacy scale and reintegration to normal living index for the second time on week 7 to observe change in fear of falling and quality of life previously measured on week 2 .

In case of medical emergencies during a TR home visit session, all providers will be given training via educational manual on when to direct patient and/or families to call 911 for emergency. Research coordinator will be in contact with study participants for the duration of the study and will relay any important issues to the rehabilitation physician who will then communicate with the rest of the team for follow-up as needed.

\section{Primary outcomes}

The primary feasibility outcomes include response rate, defined as proportion of eligible patients who consent, patient/caregiver participation with all six TR home visit interventions and acceptability of TR home visits by patients and caregivers measured via satisfaction survey. Additional data collected to plan for future randomised controlled trial are shown in figure 3 .

\section{Sample size}

A sample size of 50 is selected for this feasibility study, based on an estimate of the number of available patients who would meet the inclusion/exclusion criteria during 


\section{Stroke patients admitted to Inpatient Rehabilitation Unit at Houston based} Comprehensive Stroke Center

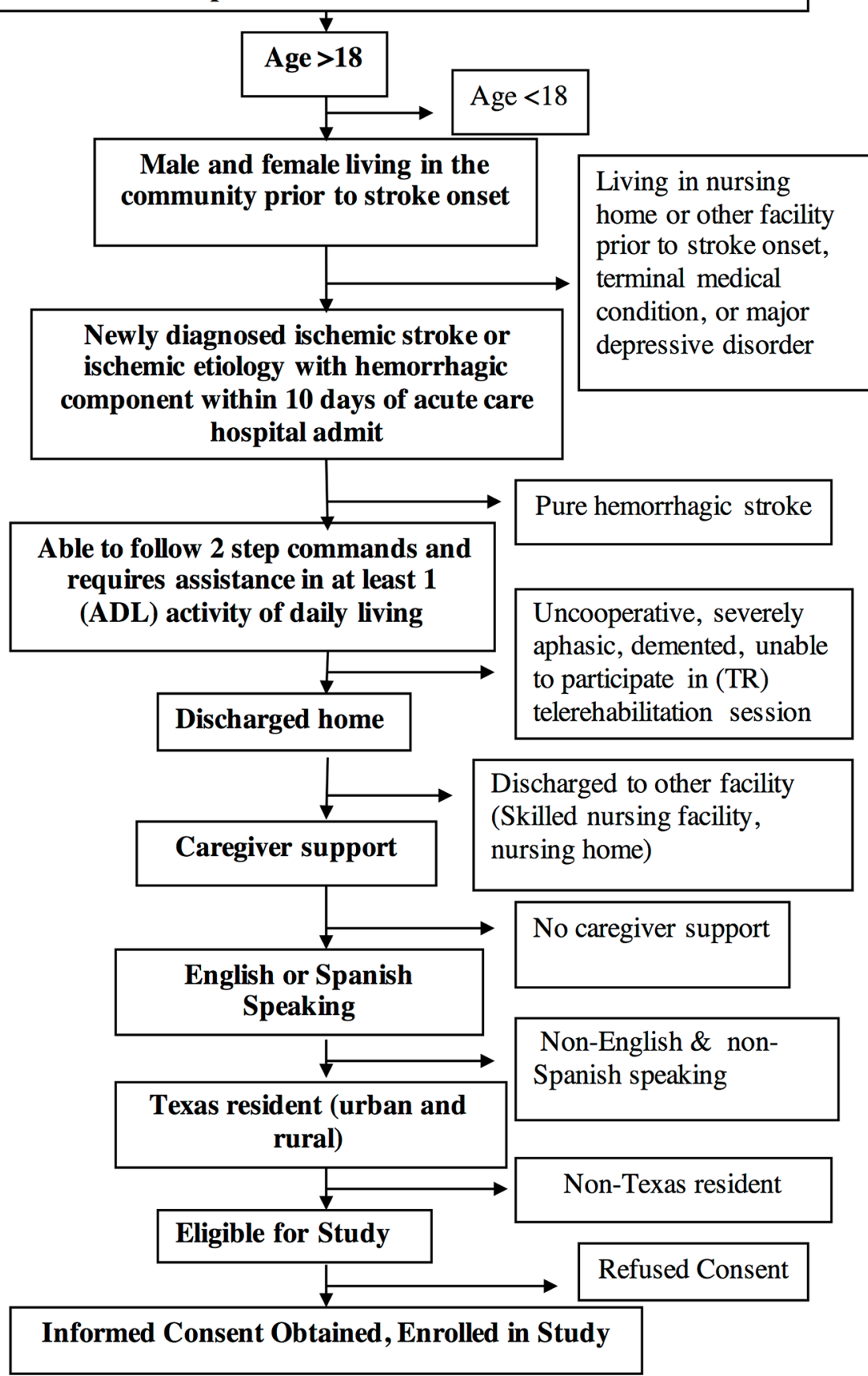

Figure 1 Study identification, inclusion and exclusion criteria.

the planned study period. Between August 2013 and August of 2014, there were 121 patients with stroke admitted to our rehab unit. Of these, 80 had ischaemic stroke and 41 had haemorrhagic stroke. Excluding those who were discharged to another facility and enrolled in other studies, we estimate we will have up to 50 eligible patients over a 24-month period.

\section{Statistical analyses}

Descriptive statistics will be performed on the enrolled patient population. Assessment of primary feasibility aims include: (1) calculating the response rate as number of eligible patients divided by number who consented; (2) calculating proportion of patients who completed all six TR home visits and various levels of compliance that is $6 / 6,5 / 6$ and so on. We will also determine if there are particular sessions that cannot be completed and (3) patient satisfaction surveys will be tallied to obtain a satisfaction score and the proportion of patients with mean satisfaction score $>70 \%$.

We will also assess factors that may affect patient/caregiver participation and programme compliance such as category/level of education, race/ethnicity, gender, 


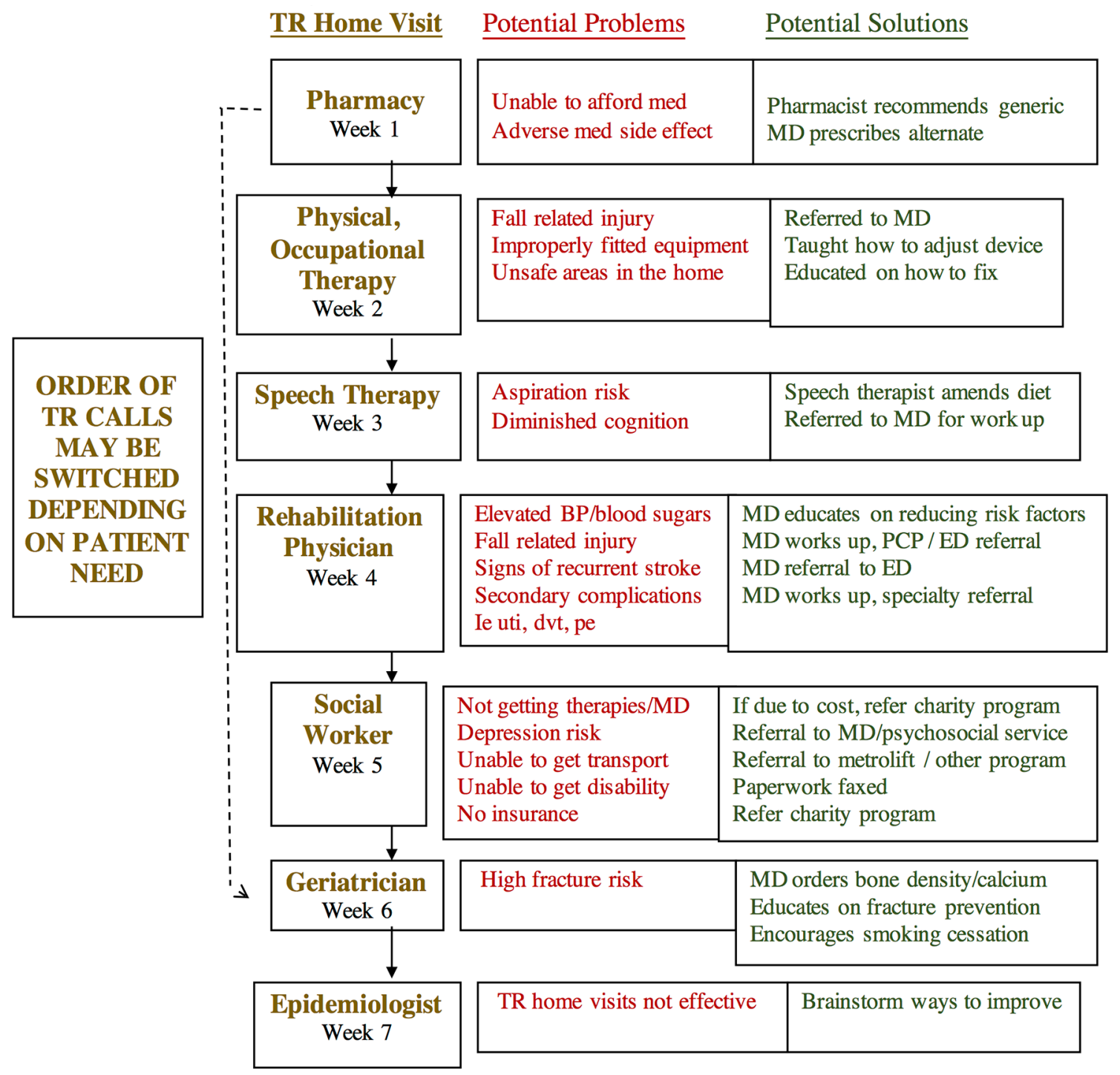

Figure 2 TR home intervention after discharge from inpatient rehabilitation. BP, blood pressure; DVT, deep venous thrombosis; ED, emergency department; MD, medical doctor; PCP, primary care physician; PE, pulmonary embolism; TR, telerehabilitation; UTI, urinary tract infection.

age, type of deficit, geographic location and insurance. These factors will be assessed as predictors in analysis of variance, with number of sessions as the continuous dependent variable. Statistically significant differences $(p<0.05)$ between levels of these variables will inform us of their impact on participation rates.

\section{Ethics and dissemination}

Ethics approval was obtained by the Institutional Review Board (IRB), the Committee on Protection of Human Subjects, Houston, Texas. They judged the study design, ethics, risks and benefits, protection of patient confidentiality, data quality control and analyses. After approval, the study was started at a Houston-based comprehensive stroke centre inpatient rehabilitation unit. Study IRB number: HSC-MS-14-0994.

A manuscript with the results of the study will be published in a peer-reviewed journal.

\section{Data monitoring}

Data quality control includes data checks on written case report form and transcription into electronic database by PI, research coordinator and data analyst. The electronic database also contains status of completed or missing reports.

\section{Risks and benefits}

As this study involves TR home visit videoconference sessions, primary risk includes maintaining confidentiality which we protect by conducting all sessions in private settings and using university IT-approved security application. Patients and caregivers accrue benefits of having the team of health professionals who worked with them on the rehabilitation unit follow-up with them during the period of transition to their home and community environment. 


\section{Data collected to assess primary feasibility outcomes and to plan sample size for future RCT}

Primary Feasibility

Outcomes:

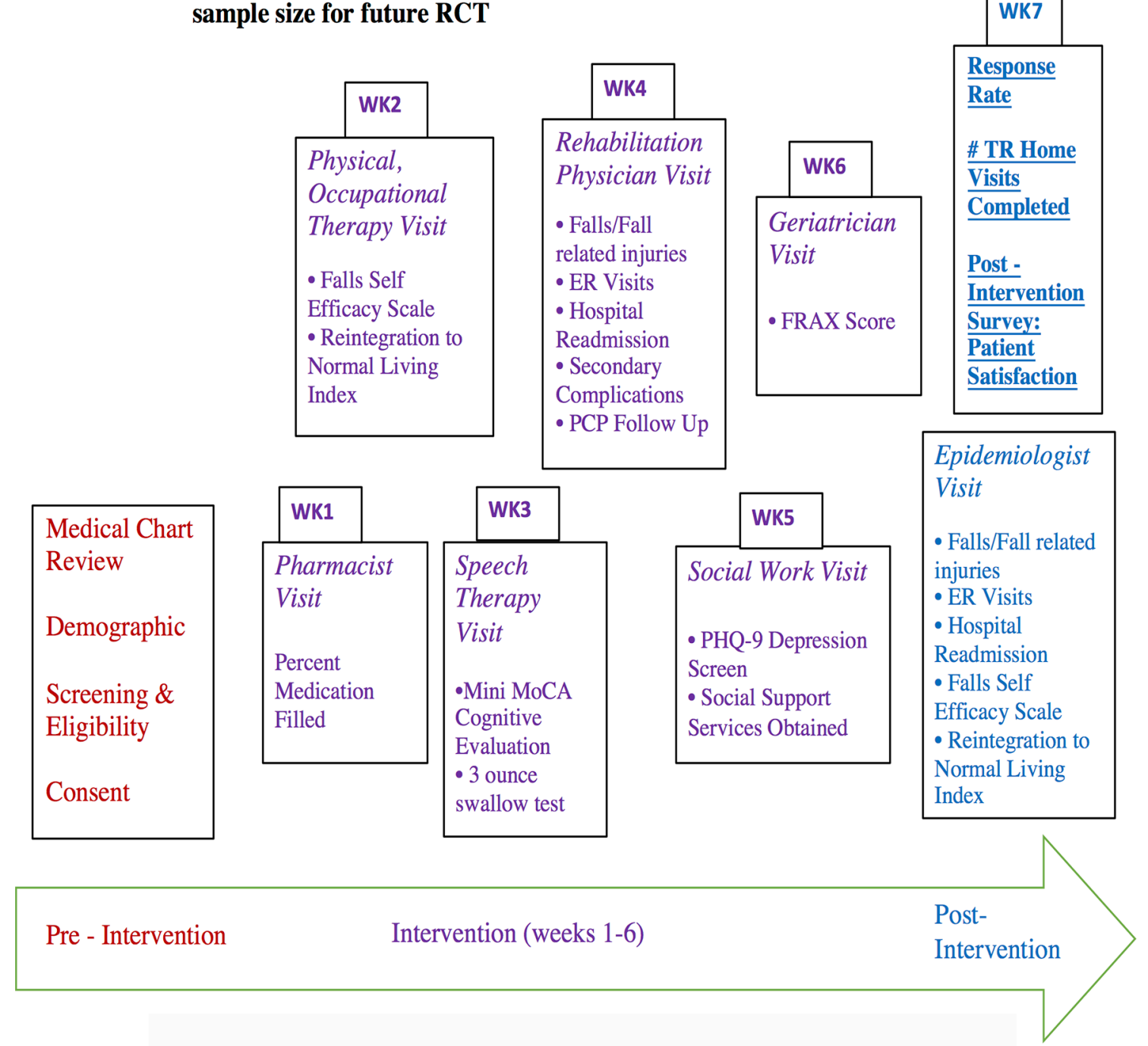

Figure 3 Telerehabilitation data collection. Response rate: number of eligible patients divided by number consented. ER, emergency room; FRAX, Fracture Risk Assessment Tool; MoCA, Montreal Cognitive Assessment; PCP, primary care physician; PHQ-9, Patient Health Questionnaire; RCT, randomised controlled trial; TR, telerehabilitation.

\section{DISCUSSION}

Transitional care of stroke rehabilitation patients is an important issue. Cognitive and physical deficits, rural location, lack of socioeconomic resources are all factors that contribute to poor physician and therapy follow-up postdischarge, leading to poorer stroke outcomes and permanent disability. The main goal of TM-SAFER feasibility study is to provide patient/caregiver access to healthcare resources, specifically rehabilitation specialists, during the critical post-discharge period. While most telerehabilitation studies focus on improving physical function in patients with chronic stroke who may have already developed secondary complications, our study focuses on educating stroke survivors and their caregivers to adopt strategies that will empower them to maintain or improve their function and quality of life before complications develop. Furthermore, most telerehabilitation studies do not incorporate a multidisciplinary team of rehabilitation specialists who may be able to address specific concerns that are unique to stroke survivors. While many clinicians may be concerned about the accuracy of evaluation via videoconferencing technology as opposed to in person visits, and possible liabilities, it is important to note that this intervention is not intended as a substitute for in person care. Rather, it is an important supplement that enhances communication and provides valuable information to patients and families struggling in the community to obtain the services they desperately need.

If the TR home visit study intervention is found to be feasible and acceptable to stroke patients, it may provide a means of making future care of stroke rehabilitation patients in the community more efficient and patient friendly. It also may provide a means for rehabilitation providers to intercept medical problems, thereby 
preventing worse outcomes related to recurrent strokes, falls, fall-related injuries and hospital readmission. Future research is needed to determine whether TR home visits are effective in improving stroke outcomes and whether policy changes on reimbursements for TR services for healthcare professionals and possibly expansion of TR services beyond state lines is warranted.

\section{Author affiliations}

${ }^{1}$ Department of Physical Medicine and Rehabilitation and Joint Appointment in Department of Neurology, University of Texas Health Science Center Houston, McGovern Medical School, Houston, Texas, USA

${ }^{2}$ Department of Internal Medicine, Center for Clinical and Translational Sciences, University of Texas Health Sciences Center Houston, McGovern Medical School, Houston, Texas, USA

${ }^{3}$ Department of Internal Medicine, Geriatric Medicine Division, University of Texas Health Sciences Center Houston, McGovern Medical School, Houston, Texas, USA ${ }^{4}$ Department of Physical Medicine and Rehabilitation, TIRR Memorial Hermann/ Memorial Hermann Rehabilitation Network, Houston, Texas, USA

${ }^{5}$ Department of Physical Medicine and Rehabilitation, TIRR Memorial Hermann/ Memorial Hermann Rehabilitation Network, University of Texas Health Science Center Houston, McGovern Medical School, Houston, Texas, USA

${ }^{6}$ Department of Neurology and Institute for Stroke and Cerebrovascular Disease, University of Texas Health Sciences Center Houston, McGovern Medical School, Houston, Texas, USA

${ }^{7}$ Pharmacy Division, TIRR Memorial Hermann, University of Texas Health Science Center Houston, McGovern Medical School, Houston, Texas, USA

${ }^{8}$ Occupational Therapy Division, Inpatient Rehabilitation, Memorial Hermann Texas Medical Center, Houston, Texas, USA

${ }^{9}$ Speech Language Pathology Division, Memorial Hermann, University of Texas Health Science Center Houston, McGovern Medical School, Houston, Texas, USA

${ }^{10}$ Division of Social Service, Memorial Hermann, University of Texas Health Science Center Houston, McGovern Medical School, Houston, Texas, USA

${ }^{11}$ Department of Neurology, University of Texas Health Science Center Houston, McGovern Medical School, Houston, Texas, USA

Contributors MMJ is principal investigator of the study and drafted the paper. All of the coauthors contributed to the study design and completion of the manuscript. Specifically, RBG contributed to data analysis considerations. MMJ, RBG, NR, KK, $M G, P S, K V$ carried out interventions and contributed to measureable outcomes included in the study. FV and AT contributed to database management. AT coordinated intervention sessions. MS, JG, SS served as senior mentors overseeing the project throughout its development. All authors were involved in the final approval of the article.

Funding Telemedicine Guided Education on Fall and Secondary Stroke Prevention is investigator initiated. Funding and sponsorship of the study is provided by The Institute for Rehabilitation and Research (TIRR), grant number 8153101-02, and Lone Star Stroke Consortium.

Competing interests None declared.

Ethics approval Institutional Review Board.
Provenance and peer review Not commissioned; externally peer reviewed.

Open Access This is an Open Access article distributed in accordance with the Creative Commons Attribution Non Commercial (CC BY-NC 4.0) license, which permits others to distribute, remix, adapt, build upon this work non-commercially, and license their derivative works on different terms, provided the original work is properly cited and the use is non-commercial. See: http://creativecommons.org/ licenses/by-nc/4.0/

(c) Article author(s) (or their employer(s) unless otherwise stated in the text of the article) 2017. All rights reserved. No commercial use is permitted unless otherwise expressly granted.

\section{REFERENCES}

1. Andersen HE, Schultz-Larsen K, Kreiner S, et al. Can readmission after stroke be prevented? Results of a randomized clinical study: a postdischarge follow-up service for stroke survivors. Stroke 2000;31:1038-45.

2. Nahab F, Takesaka J, Mailyan E, et al. Avoidable 30-day readmissions among patients with stroke and other cerebrovascular disease. Neurohospitalist 2012;2:7-11.

3. Lichtman JH, Leifheit-Limson EC, Jones SB, et al. Preventable readmissions within 30 days of ischemic stroke among medicare beneficiaries. Stroke 2013;44:3429-35.

4. Toledo FG, Ruppert K, Huber KA, et al. Efficacy of the Telemedicine for Reach, Education, Access, and Treatment (TREAT) Model for diabetes care. Diabetes Care 2014;37:e179-80.

5. McGillicuddy JW, Weiland AK, Frenzel RM, et al. Patient attitudes toward mobile phone-based health monitoring: questionnaire study among kidney transplant recipients. J Med Internet Res 2013;15:e6.

6. Clark RA, Inglis SC, McAlister FA, et al. Telemonitoring or structured telephone support programmes for patients with chronic heart failure: systematic review and meta-analysis. BMJ 2007;334:942.

7. Chumbler NR, Quigley P, Li X, et al. Effects of telerehabilitation on physical function and disability for stroke patients: a randomized, controlled trial. Stroke 2012;43:2168-74.

8. Perrin PB, Johnston A, Vogel B, et al. A culturally sensitive transition assistance program for stroke caregivers: examining caregiver mental health and stroke rehabilitation. J Rehabil Res Dev 2010;47:605-16.

9. Chumbler NR, Rose DK, Griffiths P, et al. Study protocol: homebased telehealth stroke care: a randomized trial for veterans. Trials 2010;11:74.

10. Trapl M, Enderle P, Nowotny M, et al. Dysphagia bedside screening for acute-stroke patients: the Gugging Swallowing Screen. Stroke 2007;38:2948-52.

11. Doerflinger DMC. Mental status assessment in older adults: Montreal Cognitive Assessment: MoCA Version 7.1 (Original Version). 2012 https://consultgeri.org/try-this/general-assessment/issue-3.2.pdf (accessed 15 Apr 2017).

12. Patient Health Questionnaire (PHQ-9). https://patient.info/doctor/ patient-health-questionnaire-phq-9 (accessed 15 Apr 2017).

13. Translators'/Interviewers' notes for FES-I . https://www.health.qld. gov.au/_data/assets/pdf_file/0026/426635/33346.pdf (accessed 15 Apr 2017).

14. The Reintegration to Normal Living Index (RNLI) . http://www. rehabmeasures.org/Lists/Admin\%20fields/Attachments/932/ Reintegration\%20to\%20Normal\%20Living\%20Index\%20(RNLI).pdf (accessed 15 Apr 2017).

15. (http://www.shef.ac.uk/FRAX/tool.aspx(accessed 15 April 2017)). 